\title{
A Class of p-valent Functions in the Punctured Disc
}

\section{Olubunmi A. Fadipe-Joseph ${ }^{1, *}$ and K. O. Dada ${ }^{2}$}

${ }^{1}$ Department of Mathematics, University of Ilorin, P.M.B. 1515, Ilorin, Nigeria

e-mail: famelov@unilorin.edu.ng

${ }^{2}$ Department of Mathematics, University of Ilorin, P.M.B. 1515, Ilorin, Nigeria

e-mail: dkayodeo.peniel@yahoo.com

*Corresponding author

\section{Abstract}

Motivated by Aouf differential operator, a class $F_{\lambda, p}^{n}(\alpha, \beta, \gamma)$ of p-valent functions in the punctured $\operatorname{disc} U^{*}=\{z: 0<|z|<1\}=U \backslash\{0\}$ is defined. The coefficient estimates, growth and distortion theorems for the class are obtained.

\section{Introduction}

Let $A$ denote the class of functions $g(z)$ of the form

$$
g(z)=z+\sum_{k=2}^{\infty} a_{k} z^{k}
$$

which are analytic in the open unit disc $\mathbb{U}=\{z \in \mathbb{C}:|z|<1\}$.

Let $g(z) \in A, n \in N_{0}$. Then the Ruscheweyh differential operator of the function $g(z)$ is defined as $R^{n}: A \rightarrow A$

Received: August 6, 2019; Accepted: August 24, 2019

2010 Mathematics Subject Classification: Primary 30C50; Secondary 30C45, 30C55.

Keywords and phrases: analytic function, punctured disc, differential operator, p-valent, growth and distortion.

Copyright () 2019 Olubunmi A. Fadipe-Joseph and K. O. Dada. This is an open access article distributed under the Creative Commons Attribution License, which permits unrestricted use, distribution, and reproduction in any medium, provided the original work is properly cited. 


$$
\begin{gathered}
R^{0} g(z)=g(z) \\
R^{1} g(z)=z g^{\prime}(z) \\
\vdots \\
R^{n} g(z)=z\left(R^{n-1} g(z)\right)^{\prime}+(n-1) R^{n-1}(g(z)), \quad z \in \mathbb{U} .
\end{gathered}
$$

Liu and Srivastava [1] and [2] respectively defined linear operators $L_{p}$ and $D^{n}$ on a class $\sum_{p}$ of functions which are analytic and p-valent in the punctured disc $\mathbb{U}:=\{z: z \in \mathbb{C}$ and $0<|z|<1\}=\mathbb{U} \backslash\{0\}$. Joshi and Aouf [3] considered a class consisting of regular and p-valent functions in the punctured disc with fixed second coefficients. Some properties on the class were established. Joshi and Srivastava [4] studied a certain family of meromorphically multivalent functions by using the Ruscheweyh derivative. Aouf [5] introduced a class of meromorphic multivalent functions in a punctured disc by using a differential operator to obtain some properties of the class. Logu [6] considered the works of Aouf and others thereby introduced and studied a generalized subclass of meromorphic p-valent functions using a differential operator to obtain some interesting results. Singh and Singh [7] investigated a generalized subclass of multivalent functions related to sigmoid function. Nunokawa and Sokol [8] proved some new sufficient conditions for function to be p-valent or p-valently starlike in the unit disc.

Motivated by the works of Aouf and others, let $\sum_{p}^{*}$ denote the class of functions of the form

$$
f(z)=z^{-p}+\sum_{k=1}^{\infty} a_{k} z^{k-p} \quad\left(a_{k} \geq 0 ; p \in \mathbb{N}=1,2, \ldots\right)
$$

be analytic and p-valent in the punctured disc $U^{*}=\{z: z \in C$ and $0<|z|<1$ $=U \backslash\{0\}\}$.

For $f(z) \in \sum_{p}^{*}$, define

$$
D_{\lambda, p}^{0} f(z)=f(z)
$$




$$
\begin{gathered}
D_{\lambda, p}^{1} f(z)=(1-\lambda) f(z)+\frac{\lambda}{p} z f^{\prime}(z)+\frac{2 \lambda}{z^{p}} \\
\Rightarrow D_{\lambda, p}^{1} f(z)=\frac{1}{z^{p}}+\sum_{k=1}^{\infty}\left(\frac{p-2 \lambda p+\lambda k}{p}\right) a_{k} z^{k-p} .
\end{gathered}
$$

Also,

$$
\begin{gathered}
D_{\lambda, p}^{2} f(z)=(1-\lambda) D_{\lambda, p}^{1} f(z)+\frac{\lambda z}{p}\left(D_{\lambda, p}^{1} f(z)\right)^{\prime}+\frac{2 \lambda}{z^{p}} \\
=\frac{1}{z^{p}}+\sum_{k=1}^{\infty}\left(\frac{p-2 \lambda p+\lambda k}{p}\right)^{2} a_{k} z^{k-p} \\
\vdots \\
D_{\lambda, p}^{n} f(z)=\frac{1}{z^{p}}+\sum_{k=1}^{\infty}\left(\frac{p-2 \lambda p+\lambda k}{p}\right)^{n} a_{k} z^{k-p} .
\end{gathered}
$$

\section{Main Results}

Here, the main results are discussed.

Definition 2.1. A function $f(z)$ of the form (1.2) is in the class $F_{\lambda, p}^{n}(\alpha, \beta, \gamma)$ if it satisfies

$$
\begin{gathered}
\left|\frac{z^{p+1}\left(D_{\lambda, p}^{n} f(z)\right)^{\prime}+p}{(2 \gamma-1) z^{p+1}\left(D_{\lambda, p}^{n} f(z)\right)^{\prime}+(2 \gamma \alpha-p)}\right|<\beta \\
\left(z \in U^{*} ; \alpha(0 \leq \alpha<p) ; \beta(0<\beta \leq 1) ; \gamma\left(\frac{1}{2}<\gamma \leq 1\right)\right) ; \lambda \geq 0 ; p \in \mathbb{N} ; n \in \mathbb{N}_{0} .
\end{gathered}
$$

Theorem 2.1. Let a function $f(z)$ be in the class $F_{\lambda, p}^{n}(\alpha, \beta, \gamma)$. Then

$$
\sum_{k=1}^{\infty}(k-p)\left(\frac{p-2 \lambda p+\lambda k}{p}\right)^{n}(1+2 \beta \gamma-\beta) a_{k} \leq 2 \beta \gamma(p-\alpha)
$$

for $0 \leq \alpha<p ; 0<\beta \leq 1 ; \frac{1}{2}<\gamma \leq 1 ; n \in \mathbb{N}_{0} ; p \in \mathbb{N} ; \lambda \geq 0$. 
Proof. Suppose $f(z) \in F_{\lambda, p}^{n}(\alpha, \beta, \gamma)$, then by definition

$$
\begin{gathered}
\left|\frac{z^{p+1}\left(D_{\lambda, p}^{n} f(z)\right)^{\prime}+p}{(2 \gamma-1) z^{p+1}\left(D_{\lambda, p}^{n} f(z)\right)^{\prime}+(2 \gamma \alpha-p)}\right|<\beta \\
\left(D_{\lambda, p}^{n} f(z)\right)^{\prime}=\left(\frac{1}{z^{p}}+\sum_{k=1}^{\infty} a_{k} z^{k-p}\left(\frac{p-2 \lambda p+\lambda k}{p}\right)^{n}\right)^{\prime} \\
=\frac{-p}{z^{p+1}}+\sum_{k=1}^{\infty}(k-p)\left(\frac{p-2 \lambda p+\lambda k}{p}\right)^{n} a_{k} z^{k-p-1} .
\end{gathered}
$$

Substituting (2.3), we have

$$
\begin{aligned}
& \mid \frac{z^{p+1}\left[\frac{-p}{z^{p+1}}+\sum_{k=1}^{\infty}(k-p)\left(\frac{p-2 \lambda p+\lambda k}{p}\right)^{n} a_{k} z^{k-p-1}\right]+p}{(2 \gamma-1) z^{p+1}\left[\frac{-p}{z^{p+1}}+\sum_{k=1}^{\infty}(k-p)\left(\frac{p-2 \lambda p+\lambda k}{p}\right)^{n} a_{k} z^{k-p-1}\right]+(2 \gamma \alpha-p)} \\
& =\left|\frac{-p+\sum_{k=1}^{\infty}(k-p)\left(\frac{p-2 \lambda p+\lambda k}{p}\right)^{n} a_{k} z^{k}+p}{(2 \gamma-1)\left[-p+\sum_{k=1}^{\infty}(k-p)\left(\frac{p-2 \lambda p+\lambda k}{p}\right)^{n} a_{k} z^{k}\right]+(2 \gamma \alpha-p)}\right| \\
& =\left|\frac{\sum_{k=1}^{\infty}(k-p)\left(\frac{p-2 \lambda p+\lambda k}{p}\right)^{n} a_{k} z^{k}}{2 \gamma(\alpha-p)+(2 \gamma-1) \sum_{k=1}^{\infty}(k-p)\left(\frac{p-2 \lambda p+\lambda k}{p}\right)^{n} a_{k} z^{k}}\right| \\
& <\frac{\left|\sum_{k=1}^{\infty}(k-p)\left(\frac{p-2 \lambda p+\lambda k}{p}\right)^{n} a_{k} z^{k}\right|}{\left|2 \gamma(\alpha-p)-(2 \gamma-1) \sum_{k=1}^{\infty}(k-p)\left(\frac{p-2 \lambda p+\lambda k}{p}\right)^{n} a_{k} z^{k}\right|}
\end{aligned}
$$




$$
\begin{aligned}
& =\left|\sum_{k=1}^{\infty}(k-p)\left(\frac{p-2 \lambda p+\lambda k}{p}\right)^{n} a_{k} z^{k}\right| \\
& <\beta\left|2 \gamma(\alpha-p)-(2 \gamma-1) \sum_{k=1}^{\infty}(k-p)\left(\frac{p-2 \lambda p+\lambda k}{p}\right)^{n} a_{k} z^{k}\right| \\
& \leq 2 \beta \gamma|(\alpha-p)|+\beta\left|(1-2 \gamma) \sum_{k=1}^{\infty}(k-p)\left(\frac{p-2 \lambda p+\lambda k}{p}\right)^{n} a_{k} z^{k}\right| \\
& =2 \beta \gamma|(p-\alpha)|+\beta\left|(1-2 \gamma) \sum_{k=1}^{\infty}(k-p)\left(\frac{p-2 \lambda p+\lambda k}{p}\right)^{n} a_{k}\right|\left|z^{k}\right| .
\end{aligned}
$$

Since $|z|=r<1$,

$$
\begin{aligned}
& \sum_{k=1}^{\infty}(k-p)\left(\frac{p-2 \lambda p+\lambda k}{p}\right)^{n} a_{k}-\beta(1-2 \gamma) \sum_{k=1}^{\infty}(k-p)\left(\frac{p-2 \lambda p+\lambda k}{p}\right)^{n} a_{k} \\
\leq & 2 \beta \gamma(p-\alpha) \\
= & \sum_{k=1}^{\infty}(k-p)\left(\frac{p-2 \lambda p+\lambda k}{p}\right)^{n}(1+2 \beta \gamma-\beta) a_{k} \\
\leq & 2 \beta \gamma(p-\alpha) .
\end{aligned}
$$

This implies that

$$
a_{k} \leq \frac{2 \beta \gamma(p-\alpha)}{(k-p)\left(\frac{p-2 \lambda p+\lambda k}{p}\right)^{n}(1+2 \beta \gamma-\beta)} ; k \geq 1 ; p \in \mathbb{N} ; n \in \mathbb{N}
$$

Corollary 1. A function $f(z)$ is in the class $F_{\lambda, p}^{n}(\alpha, 1,1)$ if

$$
\sum_{k=1}^{\infty}(k-p)\left(\frac{p-2 \lambda p+\lambda k}{p}\right)^{n} a_{k} \leq(p-\alpha)
$$

Corollary 2. A function $f(z)$ is in the class $F_{0, p}^{0}(\alpha, 1,1)$ if 


$$
\sum_{k=1}^{\infty}(k-p) a_{k} \leq(p-\alpha)
$$

Corollary 3. A function $f(z)$ is in the class $F_{0, p}^{0}(\alpha, \beta, 1)$ if

$$
\sum_{k=1}^{\infty}(k-p)(1+\beta) a_{k} \leq(p-\alpha) .
$$

Theorem 2.2. If the function $f(z)$ belongs to the class $F_{\lambda, p}^{n}(\alpha, \beta, \gamma)$, then for $0<|f(z)|=r<1$, we have

$$
\begin{aligned}
& \frac{1}{r^{p}}-\frac{2 \beta \gamma(p-\alpha)}{(1-p)\left(\frac{p-2 \lambda p+\lambda}{p}\right)^{n}(1+2 \beta \gamma-\beta)} r^{1-p} \\
\leq & |f(z)| \\
\leq & \frac{1}{r^{p}}+\frac{2 \beta \gamma(p-\alpha)}{(1-p)\left(\frac{p-2 \lambda p+\lambda}{p}\right)^{n}(1+2 \beta \gamma-\beta)} r^{1-p} .
\end{aligned}
$$

Proof. From Theorem 2.1,

$$
\begin{aligned}
& (1-p)\left(\frac{p-2 \lambda p+\lambda}{p}\right)^{n}(1+2 \beta \gamma-\beta) \sum_{k=1}^{\infty} a_{k} \\
\leq & \sum_{k=1}^{\infty}(k-p)\left(\frac{p-2 \lambda p+\lambda k}{p}\right)^{n}(1+2 \beta \gamma-\beta) a_{k} \\
\leq & 2 \beta \gamma(p-\alpha) .
\end{aligned}
$$

That is,

$$
\sum_{k=1}^{\infty} a_{k} \leq \frac{2 \beta \gamma(p-\alpha)}{(1-p)\left(\frac{p-2 \lambda p+\lambda}{p}\right)^{n}(1+2 \beta \gamma-\beta)}
$$


For $0<|z|=r<1$,

$$
\begin{aligned}
&|f(z)| \leq\left|\frac{1}{z^{p}}+\sum_{k=1}^{\infty} a_{k} z^{k-p}\right| \\
&=\frac{1}{r^{p}}+\sum_{k=1}^{\infty} a_{k} r^{k-p} \\
&=\frac{1}{r^{p}}+r^{1-p} \sum_{k=1}^{\infty} a_{k} \\
&|f(z)| \leq \frac{1}{r^{p}}+\frac{2 \beta \gamma(p-\alpha)}{(1-p)\left(\frac{p-2 \lambda p+\lambda}{p}\right)^{n}(1+2 \beta \gamma-\beta)} r^{1-p}
\end{aligned}
$$

and

$$
|f(z)| \leq \frac{1}{r^{p}}-\frac{2 \beta \gamma(p-\alpha)}{(1-p)\left(\frac{p-2 \lambda p+\lambda}{p}\right)^{n}(1+2 \beta \gamma-\beta)} r^{1-p}
$$

Combining (2.5) and (2.6), gives the desired result.

Theorem 2.3. Let $f(z)$ belong to the class $F_{\lambda, p}^{n}(\alpha, \beta, \gamma)$. Then for $0<|f(z)|$ $=r<1$, we have

$$
\begin{aligned}
& \frac{p}{r^{p+1}}-\frac{2 \beta \gamma(p-\alpha)}{\left(\frac{p-2 \lambda p+\lambda}{p}\right)^{n}(1+2 \beta \gamma-\beta)} r^{-p} \\
\leq & \left|f^{\prime}(z)\right| \\
\leq & \frac{p}{r^{p+1}}+\frac{2 \beta \gamma(p-\alpha)}{\left(\frac{p-2 \lambda p+\lambda}{p}\right)^{n}(1+2 \beta \gamma-\beta)} r^{-p} .
\end{aligned}
$$

Proof. In view of Theorem 2.1, 


$$
\sum_{k=1}^{\infty} a_{k} \leq \frac{2 \beta \gamma(p-\alpha)}{(1-p)\left(\frac{p-2 \lambda p+\lambda}{p}\right)^{n}(1+2 \beta \gamma-\beta)}
$$

Thus,

$$
\begin{gathered}
\left|f^{\prime}(z)\right| \leq\left|\frac{-p}{z^{p+1}}\right|+\left|\sum_{k=1}^{\infty} a_{k} z^{-p}\right| \\
\left|f^{\prime}(z)\right| \leq \frac{p}{r^{p+1}}+\frac{2 \beta \gamma(p-\alpha)}{\left(\frac{p-2 \lambda p+\lambda}{p}\right)^{n}(1+2 \beta \gamma-\beta)} r^{-p} .
\end{gathered}
$$

Similarly,

$$
\left|f^{\prime}(z)\right| \geq \frac{p}{r^{p+1}}-\frac{2 \beta \gamma(p-\alpha)}{\left(\frac{p-2 \lambda p+\lambda}{p}\right)^{n}(1+2 \beta \gamma-\beta)} r^{-p}
$$

From (2.8) and (2.9), the result follows.

\section{References}

[1] J. L. Liu and H. M. Srivastava, A linear operator and associated families of meromorphically multivalent functions, J. Math. Anal. Appl. 259 (2001), 566-581. https://doi.org/10.1006/jmaa.2000.7430

[2] J. L. Liu and H. M. Srivastava, Subclasses of meromorphically multivalent functions associated with a certain linear operator, Math. Comput. Modelling 39(1) (2004), 35-44. https://doi.org/10.1016/S0895-7177(04)90504-3

[3] S. B. Joshi and M. K. Aouf, Meromorphic multivalent functions with positive and fixed second coefficients, Kyungpook Math. J. 35(1995), 163-169.

[4] S. B. Joshi and H. M. Srivastava, A certain family of meromorphically multivalent functions, Comput. Math. Appl. 38(3-4) (1999), 201-211. https://doi.org/10.1016/S0898-1221(99)00194-7

[5] M. K. Aouf, A class of meromorphic multivalent functions with positive coefficients, Taiwanese J. Math. 12(9) (2008), 2517-2533. https://doi.org/10.11650/twjm/1500405193 
[6] S. Logu, Some subclasses of meromorphic p-valent functions with positive coefficients defined on the punctured disc using a differential operator, Int. J. Multidiscip. Educ. Res. 1 (2016), 4-17.

[7] G. Singh and G. Singh, A generalized subclass of multivalent functions related to sigmoid function, J. Ultra Sci. Phys. Sci. 30(3) (2018), 164-171.

https://doi.org/10.22147/jusps-A/300301

[8] M. Nunokawa and J. Sokol, On multivalent starlike functions and Ozaki condition, Complex Var. Elliptic Equ. 64(1) (2019), 78-92.

https://doi.org/10.1080/17476933.2017.1419209 